\title{
Two-dimensional charge order stabilized in clean polytype heterostructures
}

Suk Hyun Sung ${ }^{1}$, Noah Schnitzer ${ }^{2}$, Steve Novakov ${ }^{1}$, Ismail El Baggari ${ }^{3}$, Xiangpeng Luo ${ }^{1}$, Jiseok Gim ${ }^{1}$, Nguyen $\mathrm{Vu}^{1}$, Zidong $\mathrm{Li}^{1}$, Todd Brintlinger ${ }^{4}$, Yu Liu ${ }^{5}$, Wenjian Lu ${ }^{5}$, Yuping Sun ${ }^{5}$, Parag Deotare ${ }^{1}$, Kai Sun $^{1}$, Liuyan Zhao ${ }^{1}$, Lena Kourkoutis ${ }^{6}$, John Heron ${ }^{1}$ and Robert Hovden ${ }^{7}$

${ }^{1}$ University of Michigan, United States, ${ }^{2}$ Department of Materials Science and Engineering, Cornell University, United States, ${ }^{3}$ The Rowland Institute at Harvard University, United States, ${ }^{4}$ U.S. Naval Research Laboratory, United States, ${ }^{5}$ Chinese Academy of Sciences, United States, ${ }^{6}$ School of Applied and Engineering Physics, Cornell University, United States, ${ }^{7}$ Department of Materials Science and Engineering, University of Michigan, United States

Strong evidence suggests that transformative correlated electron behavior may exist only in unrealized clean-limit 2D 1T-TaS 2 [1]. Unfortunately, experiment [2,3] and theory [4] suggest that extrinsic disorder in free standing 2D layers impedes correlation-driven quantum behavior. Here we leverage in-situ TEM (transmission electron microscopy), atomic resolution STEM (scanning TEM) and 4D-STEM (scanning electron diffraction) to demonstrate a new route to realizing fragile 2D quantum states through epitaxial polytype engineering of van der Waals materials [5]. More specifically, we show the long-range ordered commensurate (C) CDW expected at low temperatures $(200 \mathrm{~K})$ is stable at room temperature in 2D [5]. The isolation of truly 2D CDWs between metallic layers stabilizes C-CDWs and lifts the coupling between neighboring CDW layers to restore mirror symmetries via interlayer CDW twinning. The twinned commensurate (tC)-CDW phase reported herein has distinct out-of-plane charge order-illustrated in Figure 1a. 2D CDWs reside within octahedral (Oc) layers sparsely interdigitated between metallic prismatic (Pr) layers. Moreover, the truly 2D CDWs stabilizes with commensurate long-range order at room-temperature but expected only at low-temperatures.

4D-STEM of the tC-CDW phase maps both degenerate twin CDW states, $\alpha-C$ (blue) or $\beta-\mathrm{C}$ (red) (Fig 1b, c) over microns of area to reveal a uniform co-existence of both twins when viewed in projection out-ofplane (Fig. 1e, f). In this way, the local CDW structure was measured at $\sim 4.6 \mathrm{~nm}$ resolution and across $>1$ $\mu \mathrm{m}$ fields of view. Figure $1 \mathrm{~d}$ shows the position averaged convergent beam electron diffraction pattern $(0.55 \mathrm{mrad}$ semi-convergence angle, $80 \mathrm{keV})$ of the tC-CDW phase at room temperature with $\alpha, \beta$ superlattice peaks annotated (blue, red). Regularly spaced superlattice peaks and bright first order superlattice peaks are characteristic of C-CDWs and match the tC-CDW peaks.

The tC-CDW phase herein results from interdigitated polytypes synthesized by precise thermal cyclinga process summarized by the in-situ selected area electron diffraction (SAED) in Figure 2a. Initially, an exfoliated flake of $1 \mathrm{~T}-\mathrm{TaS}_{2}$ hosts the disordered CDW (i.e. nearly commensurate or NC) (Fig. 2a, left) at room temperature with diffuse first-order superlattice peaks (cyan circles) and sharp second order superlattice peaks (cyan triangle). $1 \mathrm{~T}-\mathrm{TaS}_{2}$ is heated past the reversible phase transition ( $\mathrm{T}_{\mathrm{NC}-\mathrm{IC}} \sim 352 \mathrm{~K}$ ) into the disordered incommensurate CDW state, with characteristic azimuthally diffused superlattice spots (Fig. 2a, right). Heating continues above the polytype transition temperature $(\sim 600 \mathrm{~K})$ where it remains for several minutes. Upon cooling, the system does not return to the expected NC-CDW but instead enters a tC-CDW state with sharp, evenly spaced commensurate first and second order superlattice peaks duplicated with mirror symmetry $(\alpha, \beta)$ (Fig. 2a, bottom). 
Atomic resolution cross-section images of pristine and heat-treated samples (Fig. $2 \mathrm{~b}$ and c, respectively) measured by high-angle annular dark-field (HAADF)-STEM reveal the interdigitated polytypic heterostructure. Interdigitation isolates monolayers of Oc coordination that host 2D-CDWs in a clean, defect-free environment of metallic Pr layers. This result is radically different from previous reports where free standing ultra-thin $1 \mathrm{~T}-\mathrm{TaS}_{2}$ degrades long-range order [6] and broadens the NC-CDW phase by lowering TCCDW $[3,7,8]$.

Electronic measurement of the polytypic heterostructure with interdigitated CDWs reveals a direct $\mathrm{tC} \leftrightharpoons \mathrm{IC}$ transition at $350 \mathrm{~K}$ and removal of the disordered NC-CDW phase. Figure $2 \mathrm{~d}$ shows in-plane resistance vs. temperature measurements of pristine $1 \mathrm{~T}$ (pink) and heat-treated polytypic heterostructure (blue) are drastically different. The heterostructure features only one metal-insulator transition at $350 \mathrm{~K}$, whereas

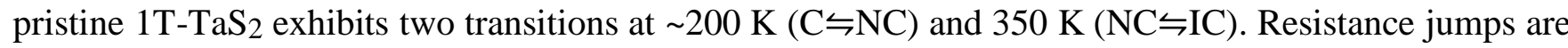
a signature of emergent $\mathrm{CDW}$ order. At low temperature the metallic Pr-layers dominate in-plane conduction since the resistance is expected to monotonically decrease as observed in bulk material [9]; however the critical temperatures remain clearly visible.

In summary, latent CDWs with long-range order were stabilized at room-temperature by 2D confinement in a clean-limit using engineered interdigitated polytypes of $\mathrm{TaS}_{2}$. The metallic layers isolate each $2 \mathrm{D}$ $\mathrm{CDW}$ to restore twin degeneracy giving rise to an out-of-plane tC-CDW phase. 4D-STEM proved invaluable for mapping CDW domains across large fields-of-view $(\sim 1 \mu \mathrm{m})$ to confirm twin structure while atomic resolution HAADF-STEM revealed the 2D CDW layers within metallic layers.
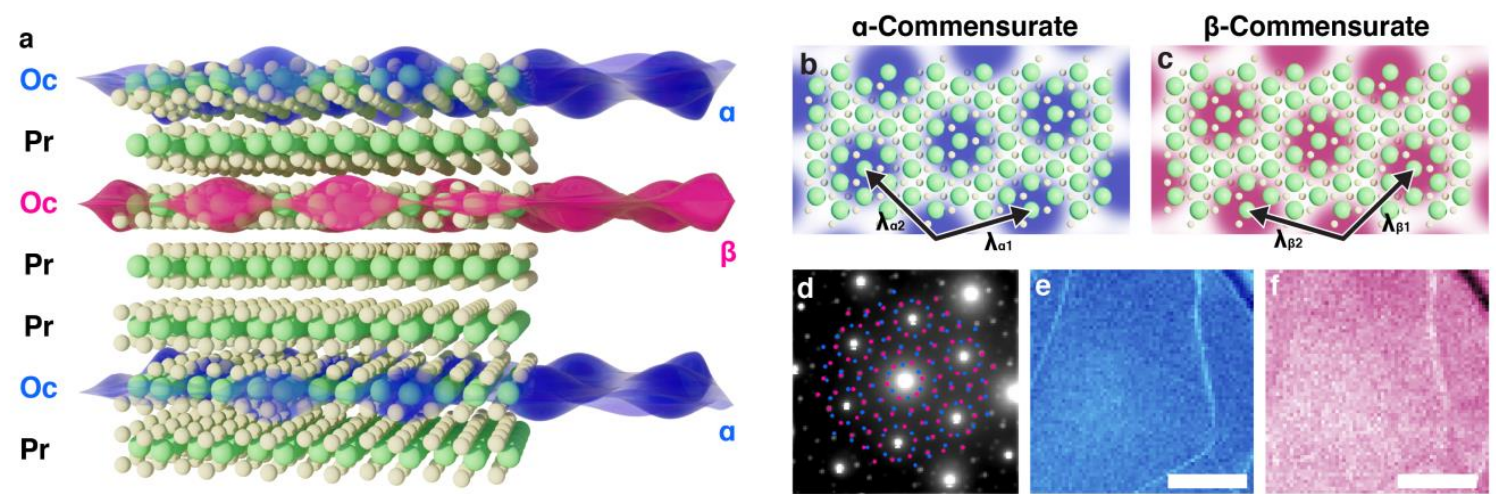

Figure 1. Figure 1 | Twinned, commensurate CDW at room temperature in ultrathin TaS2. a) Schematic illustration of roomtemperature, out-of-plane twinned, commensurate CDW in 1T-TaS2. Blue and red overlays represent CDW twins within octahedrally coordinated TaS2. Metallic prismatic polytypes isolate octahedral layers to stabilize tC-CDWs. b, c) Twin superlattice structure illustrated for $\alpha$ and $\beta$ C-CDW, respectively. d) Average diffraction pattern of twinned, C-CDW state over $(870 \mathrm{~nm}) 2$ field-of-view reveals two sets of superlattice peaks (marked with blue and red). e, f) Nano beam diffraction imaging from each set of superlattice peaks maps the coexistence of both CDW twins-expected for twinning out-of-plane. Scale bar is $300 \mathrm{~nm}$. Figure reproduced from [5]. 

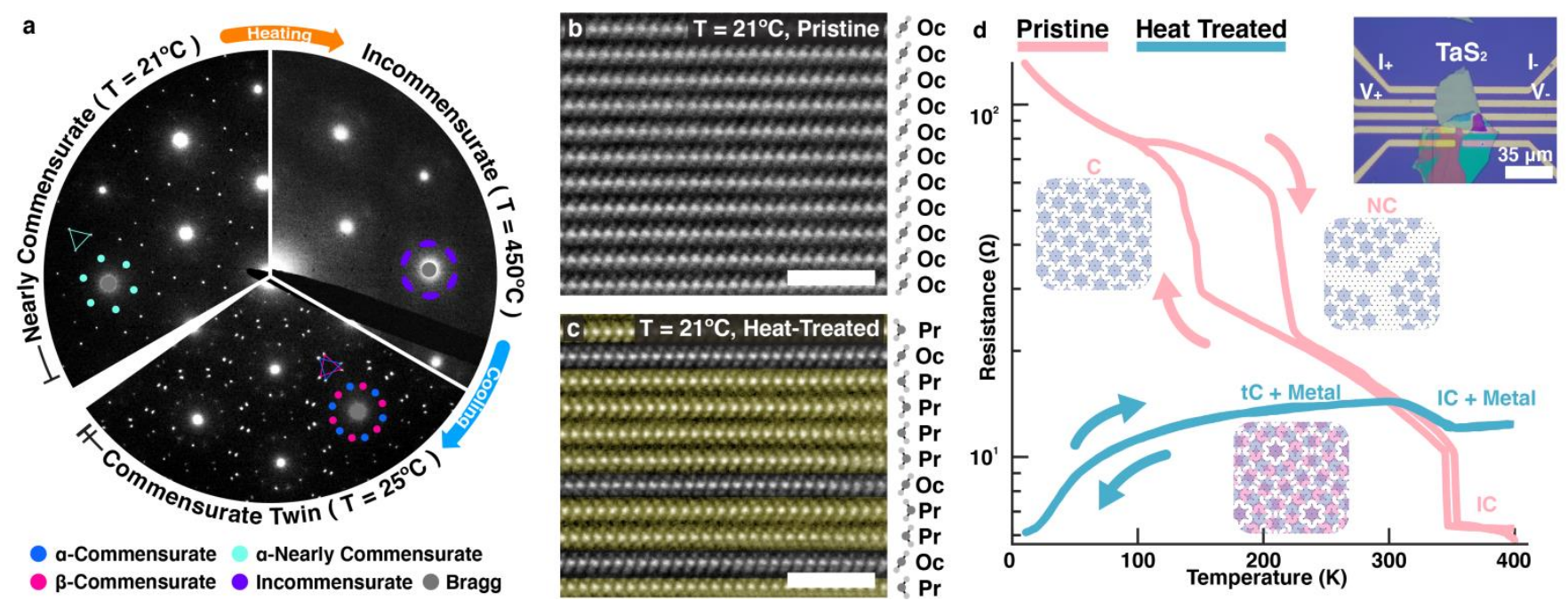

Figure 2. Figure $2 \mid$ Polytype isolation forms 2D CDW layers. a) Pristine 1T-TaS2 at room temperature hosts NC-CDW (left). Upon heating the NC phase gives way to IC-CDW (right) at $\sim 80^{\circ} \mathrm{C}$; the transition is normally reversible. Strikingly, heating above $\sim 350^{\circ} \mathrm{C}$ then cooling stabilizes tC-CDW (bottom) b-c) Atomic resolution cross-sectional HAADF-STEM of b) pristine and c) heat-treated TaSxSe2-x confirms polytypic transformation. After treatment, prismatic (Pr) layers encapsulate monolayers of octahedral (Oc) layers. Scale bar is $2 \mathrm{~nm}$. A selenium doped sample was imaged to enhance chalcogen visibility. d) 4-point in-plane resistance measurement as function of temperature for pristine bulk (pink) and heat-treated (blue) TaS2. Pristine samples show two jumps in resistance for $\mathrm{C} \leftrightharpoons \mathrm{NC}$ and $\mathrm{NC} \leftrightharpoons \mathrm{IC}$, whereas the heat treated polytypic heterostructures only feature a single, reversible tC $\leftrightharpoons \mathrm{IC}$ transition at $\sim 350 \mathrm{~K}$ corresponding to the enhanced critical temperature for CDW commensuration and disappearance of the NC-CDW. Metallic Pr-layers dominate the overall trend of the resistance measurement, however, the single jump above room-temperature is a distinct feature of the $\mathrm{tC}$-CDW. Inset) Optical image of the nanofabricated device.

\section{References}

[1] K. Law and P. Lee, Proc. Natl. Acad. Sci., 114:6996-7000 (2017).

[2] Y. Yu et al., Nat. Nanotechnol., 10:270-276 (2015).

[3] A. Tsen et al., Proc. Natl. Acad. Sci., 112:15054-15059 (2015).

[4] L. Nie et al., Proc. Natl. Acad. Sci., 111:7980-7985 (2014).

[5] S. H. Sung et al., Arxiv, 2102.09079 (2021).

[6] D. Sakabe et al., npj Quantum Mater., 2:22 (2017).

[7] M. Yoshida et al., Sci. Rep., 4:7302 (2014).

[8] Y. Yu et al., Nat. Nanotechnol., 10:270-276 (2015).

[9] A. H. Thompson et al., Phys. Rev. B., 5:2811-2816 (1972). 